\title{
New multiplicity of positive solutions for some class of nonlocal problems
}

\section{Zhigao Shi ${ }^{1}$ and Xiaotao Qian ${ }^{2 *}$ (D)}

\author{
"Correspondence: \\ qianxiaotao1984@163.com \\ ${ }^{2}$ Department of Basic Teaching and \\ Research, Yango University, Fuzhou, \\ 350015, P.R. China \\ Full list of author information is \\ available at the end of the article
}

\section{Abstract}

In this paper, we study the following nonlocal problem:

$$
\begin{cases}-\left(a-b \int_{\Omega}|\nabla u|^{2} d x\right) \Delta u=\lambda|u|^{a-2} u, & x \in \Omega, \\ u=0, & x \in \partial \Omega,\end{cases}
$$

where $\Omega$ is a smooth bounded domain in $\mathbb{R}^{N}$ with $N \geq 3, a, b>0,1<q<2$ and $\lambda>0$ is a parameter. By virtue of the variational method and Nehari manifold, we prove the existence of multiple positive solutions for the nonlocal problem. As a co-product of our arguments, we also obtain the blow-up and the asymptotic behavior of these solutions as $b \searrow 0$.

MSC: $35 J 20 ; 35 J 60$

Keywords: Variational method; Nonlocal problem; Multiple positive solutions; Blow up

\section{Introduction and main results}

In this paper, we are concerned with the multiplicity of positive solutions for the nonlocal problem

$$
\begin{cases}-\left(a-b \int_{\Omega}|\nabla u|^{2} d x\right) \Delta u=\lambda|u|^{q-2} u, & x \in \Omega, \\ u=0, & x \in \partial \Omega,\end{cases}
$$

where $\Omega$ is a smooth bounded domain in $\mathbb{R}^{N}(N \geq 3), a, b>0,1<q<2$ and $\lambda>0$ is a parameter.

In the past two decades, the following Kirchhoff type problem on a bounded domain

$$
\begin{cases}-\left(a+b \int_{\Omega}|\nabla u|^{2} d x\right) \Delta u=f(x, u), & x \in \Omega, \\ u=0, & x \in \partial \Omega,\end{cases}
$$

has attracted great attention of many researchers. The Kirchhoff type problem is often viewed as nonlocal due to the presence of the term $b \int_{\Omega}|\nabla u|^{2} d x$ which implies that such a

(c) The Author(s) 2021. This article is licensed under a Creative Commons Attribution 4.0 International License, which permits use, sharing, adaptation, distribution and reproduction in any medium or format, as long as you give appropriate credit to the original author(s) and the source, provide a link to the Creative Commons licence, and indicate if changes were made. The images or other third party material in this article are included in the article's Creative Commons licence, unless indicated otherwise in a credit line to the material. If material is not included in the article's Creative Commons licence and your intended use is not permitted by statutory regulation or exceeds the permitted use, you will need to obtain permission directly from the copyright holder. To view a copy of this licence, visit http://creativecommons.org/licenses/by/4.0/. 
problem is no longer a pointwise identity. By using the variational method, there are many interesting results of positive solutions to (1.2), see e.g. $[1,4,5,9,11]$ and the references therein.

If we replace $b \int_{\Omega}|\nabla u|^{2} d x$ with $-b \int_{\Omega}|\nabla u|^{2} d x$, then (1.2) turns out to be the following new nonlocal one:

$$
\begin{cases}-\left(a-b \int_{\Omega}|\nabla u|^{2} d x\right) \Delta u=f(x, u), & x \in \Omega, \\ u=0, & x \in \partial \Omega .\end{cases}
$$

This kind of problem involving negative nonlocal term not only presents some interesting difficulties different from Kirchhoff type problem but also has its own physical and mechanical motivation, see [8, 14]. Yin and Liu [16] considered problem (1.3) when $f(x, u)=|u|^{p-2} u$ with $2<p<2^{*}$ and showed the existence of two nontrivial solutions. Based on [16], Wang and Yang [15] further obtained the existence of infinitely many signchanging solutions. In [17], the authors extended the results of [16] to a general case of nonlinear terms. For $f(x, u)=\lambda|u|^{-\gamma}$ with $0<\gamma<1$, [6] got the multiplicity of positive solutions to (1.3). In [10], we proved that problem (1.3) possesses at least one positive solution when $N=3, f(x, u)=\lambda f(x)|u|^{p-2} u$ with $3<p<4$ and $f(x) \in L^{\frac{6}{6-p}}(\Omega)$ may change sign. In particular, Duan et al. [3] and Lei et al. [7] proved that there exists $\lambda_{*}>0$ such that, for each $\lambda \in\left(0, \lambda_{*}\right)$, problem (1.1) has two positive solutions by using the minimization argument and the mountain pass theorem.

From the works described before, it is important and interesting to ask whether the multiplicity of positive solutions to problem (1.1) can be established by other methods? In the present paper, we shall give an affirmative answer. The main technique applied here is a separation argument for the Nehari-type set of problem (1.1), which has been firstly introduced by Tarantello [13] and later refined by Sun and $\mathrm{Li}$ [12].

Let $H:=H_{0}^{1}(\Omega)$ and $L^{s}(\Omega)$ be the standard Sobolev spaces endowed with the standard norms $\|\cdot\|$ and $|\cdot|_{p}$, respectively. Denote by $\rightarrow$ and $\rightarrow$ the strong and weak convergence, respectively. We use $o_{n}(1)$ to denote a quantity such that $o_{n}(1) \rightarrow 0$ as $n \rightarrow \infty$. $C$ and $C_{i}$ denote various positive constants which may vary from line to line. We say that $I \in C^{1}(H, \mathbb{R})$ satisfies the Palais-Smale condition at level $c \in \mathbb{R}\left((\mathrm{PS})_{c}\right.$ in short) if any sequence $\left\{u_{n}\right\} \subset H$ such that $I\left(u_{n}\right) \rightarrow c$ and $I^{\prime}\left(u_{n}\right) \rightarrow 0$ in $H^{-1}$ as $n \rightarrow \infty$ has a convergent subsequence. $S$ denotes the best constant in the Sobolev embedding $H \hookrightarrow L^{2^{*}}(\Omega)$, that is,

$$
S=\inf _{u \in H \backslash\{0\}} \frac{\int|\nabla u|^{2} d x}{\left(\int|u|^{2^{*}} d x\right)^{2 / 2^{*}}}>0 .
$$

Associated with problem (1.1), we define the energy functional

$$
I_{b}(u)=\frac{a}{2}\|u\|^{2}-\frac{b}{4}\|u\|^{4}-\frac{\lambda}{q} \int_{\Omega}|u|^{q} d x .
$$

Then $I_{b} \in C^{1}(H, \mathbb{R})$. Recall that a function $u \in H$ is called a weak solution to (1.1) if, for any $\phi \in H$, there holds

$$
\left(a-b\|u\|^{2}\right) \int_{\Omega} \nabla u \nabla \phi d x-\lambda \int_{\Omega}|u|^{q-2} u \phi d x=0 .
$$


Define the Nehari type set of (1.1)

$$
\Lambda_{b}=\left\{u \in H:\left\langle I_{b}^{\prime}(u), u\right\rangle=0\right\}=\left\{u \in H: a\|u\|^{2}-b\|u\|^{4}=\lambda \int_{\Omega}|u|^{q} d x\right\}
$$

and then decompose $\Lambda_{b}$ into three subsets:

$$
\begin{aligned}
& \Lambda_{b}^{0}=\left\{u \in \Lambda_{b}: a(2-q)\|u\|^{2}-b(4-q)\|u\|^{4}=0\right\}, \\
& \Lambda_{b}^{+}=\left\{u \in \Lambda_{b}: a(2-q)\|u\|^{2}-b(4-q)\|u\|^{4}>0\right\}, \\
& \Lambda_{b}^{-}=\left\{u \in \Lambda_{b}: a(2-q)\|u\|^{2}-b(4-q)\|u\|^{4}<0\right\} .
\end{aligned}
$$

It is important to notice that there exists a norm gap in $\Lambda_{b}$ :

$$
\|\tilde{u}\|^{2}>\frac{a(2-q)}{b(4-q)}>\|u\|^{2} \quad \text { for all } u \in \Lambda_{b}^{+}, \tilde{u} \in \Lambda_{b}^{-}
$$

Set

$$
T_{b}=\frac{2(2-q)}{(4-q)^{2}} \frac{a^{\frac{4-q}{2}}}{b^{\frac{2-q}{2}}} \frac{S^{\frac{q}{2}}}{|\Omega|^{\frac{2^{*}-q}{2^{*}}}} .
$$

Our main results are as follows.

Theorem 1.1 Assume that $\lambda \in\left(0, T_{b}\right)$, then problem (1.1) has at least two positive solutions $u_{*} \in \Lambda_{b}^{+}, \tilde{u}_{*} \in \Lambda_{b}^{-}$with $\left\|u_{*}\right\|<\left\|\tilde{u}_{*}\right\|$.

Moreover, as a by-product of our arguments, we regard $b$ as a parameter and obtain the blow-up behavior of the solution $\tilde{u}_{b} \in \Lambda_{b}^{-}$and the asymptotic behavior of the other one $u_{b} \in \Lambda_{b}^{+}$of problem (1.1) as $b \searrow 0$. Namely, we have the following theorem.

Theorem 1.2 Assume that $\left\{b_{n}\right\}$ is a sequence satisfying $b_{n} \searrow 0$ as $n \rightarrow \infty$. Then there exists a subsequence, still denoted by $\left\{b_{n}\right\}$, such that

(i) $\left\|\tilde{u}_{b_{n}}\right\| \rightarrow \infty$ as $n \rightarrow \infty$.

(ii) $u_{b_{n}} \rightarrow u_{0}$ in $H$ as $n \rightarrow \infty$, where $u_{0}$ is a positive solution of the problem

$$
\begin{cases}-a \Delta u=\lambda|u|^{q-2} u, & x \in \Omega \\ u=0, & x \in \partial \Omega\end{cases}
$$

Remark 1.3 Compared with [3, 7], we adapt a new method to show the existence and multiplicity of positive solutions to problem (1.1). In particular, we obtain the blow-up and the asymptotic behavior of these solutions. As far as we know, such phenomena about the solutions to (1.1) are first observed, which reveals some relationship between the nonlocal problem (1.1) and the classical semilinear problem (1.5).

The paper is organized as follows. In Sect. 2, we present some preliminaries. Sections 3 and 4 are devoted to the proofs of Theorems 1.1 and 1.2, respectively. 


\section{Preliminaries}

Lemma 2.1 Let $\lambda \in\left(0, T_{b}\right)$. Then $\Lambda_{b}^{ \pm} \neq \emptyset$ and $\Lambda_{b}^{0}=\{0\}$.

Proof For any $u \in H, u \neq 0$, we define

$$
h(t)=a t^{2-q}\|u\|^{2}-b t^{4-q}\|u\|^{4}, \quad \forall t>0 .
$$

It is easy to see that $g(t)$ attains its maximum value at $t_{\max }=\left[\frac{a(2-q)}{b(4-q)\|u\|^{2}}\right]^{1 / 2}$ with

$$
h\left(t_{\max }\right)=\frac{2(2-q) a^{\frac{4-q}{2}}}{(4-q)^{2}} \frac{2-q}{b^{\frac{2-q}{2}}}\|u\|^{q} .
$$

We note that, by Hölder's inequality, for $\lambda \in\left(0, T_{b}\right)$, there holds

$$
\lambda \int_{\Omega}|u|^{q} d x \leq \lambda|\Omega|^{2^{*}-q} S^{-q / 2}\|u\|^{q}<h\left(t_{\max }\right) .
$$

It follows that there are two and only two positive constants $t^{+}=t^{+}(u)$ and $t^{-}=t^{-}(u)$ such that

$$
h\left(t^{+}\right)=\lambda \int_{\Omega}|u|^{q} d x=h\left(t^{-}\right) \text {and } \quad h^{\prime}\left(t^{+}\right)<0<h^{\prime}\left(t^{-}\right) .
$$

Equivalently, we obtain $t^{+} u \in \Lambda_{b}^{-}$and $t^{-} u \in \Lambda_{b}^{+}$.

Next, we prove that $\Lambda_{b}^{0}=\{0\}$. Arguing by contradiction, we assume that there exists $w \in \Lambda_{b}^{0}$ satisfying $w \neq 0$. Then we have $a(2-q)\|w\|^{2}-b(4-q)\|w\|^{4}=0$. This yields $b\|w\|^{2}=$ $\frac{a(2-q)}{4-q}$. For $\lambda \in\left(0, T_{b}\right)$, it follows from $w \in \Lambda_{b}$ and Hölder's inequality that

$$
\begin{aligned}
0 & =a\|w\|^{2}-b\|w\|^{4}-\lambda \int_{\Omega}|w|^{q} d x \\
& \geq \frac{2 a}{4-q}\|w\|^{2}-\lambda|\Omega|^{\frac{2^{*}-q}{2^{*}}} S^{-q / 2}\|w\|^{q} \\
& =\|w\|^{q}\left[\frac{2(2-q)}{(4-q)^{2}} \frac{a^{\frac{4-q}{2}}}{b^{\frac{2-q}{2}}}\left(\frac{4-q}{2-q}\right)^{\frac{q}{2}}-\lambda|\Omega|^{\frac{2^{*}-q}{2^{*}}} S^{\frac{-q}{2}}\right]>0,
\end{aligned}
$$

which makes no sense. This ends the proof.

Lemma 2.2 Given $u \in \Lambda_{b}^{ \pm}$, there exist $\rho_{u}>0$ and a differential function $g_{\rho_{u}}: B_{\rho_{u}}(0) \rightarrow \mathbb{R}^{+}$ defined for $w \in H, w \in B_{\rho_{u}}(0)$ satisfying

$$
g_{\rho_{u}}(0)=1, \quad g_{\rho_{u}}(w)(u-w) \in \Lambda_{b}^{ \pm},
$$

and

$$
\left\langle g^{\prime}(0), \phi\right\rangle=\frac{\left(2 a-4 b\|u\|^{2}\right) \int_{\Omega} \nabla u \nabla \phi d x-q \lambda \int_{\Omega}|u|^{q-2} u \phi d x}{a(2-q)\|u\|^{2}-b(4-q)\|u\|^{4}} .
$$


Proof We only give the proof for the case $u \in \Lambda_{b}^{-}$. In a similar way, one can prove the other case $u \in \Lambda_{b}^{+}$. Fix $u \in \Lambda_{b}^{-}$and define $F: \mathbb{R}^{+} \times H \rightarrow \mathbb{R}$ by

$$
F(t, w)=a t\|u-w\|^{2}-b t^{3}\|u-w\|^{4}-\lambda t^{q-1} \int_{\Omega}|u-w|^{q} d x .
$$

By $u \in \Lambda_{b}^{-} \subset \Lambda_{b}$, we easily see that $F(1,0)=0$ and

$$
F_{t}(1,0)=a(2-q)\|u\|^{2}-b(4-q)\|u\|^{4}<0 .
$$

Then, we are able to use the implicit function theorem for $F$ at the point $(1,0)$ and get $\bar{\rho}>0$ and a differential functional $g=g(w)>0$ defined for $w \in H,\|w\|<\bar{\rho}$ such that

$$
g(0)=1, \quad g(w)(u-w) \in \Lambda_{b}, \quad \forall w \in H,\|w\|<\bar{\rho} .
$$

Thanks to the continuity of $g$, we can take $\rho>0$ possibly smaller $(\rho<\bar{\rho})$ such that, for any $w \in H,\|w\|<\rho$, there holds

$$
g(w)(u-w) \in \Lambda_{b}^{-}
$$

Moreover, for any $\phi \in H, r>0$, it follows from

$$
\begin{aligned}
F(1,0 & +r \phi)-F(1,0) \\
= & a\|u-r \phi\|^{2}-b\|u-r \phi\|^{4}-\lambda \int_{\Omega}|u-r \phi|^{q} d x-a\|u\|^{2}+b\|u\|^{4}+\lambda \int_{\Omega}|u|^{p} d x \\
= & -a \int_{\Omega}\left(2 r \nabla u \nabla \phi-r^{2}|\nabla \phi|^{2}\right) d x-\lambda \int_{\Omega}\left(|u-r \phi|^{q}-|u|^{q}\right) d x \\
& +b\left[2 \int_{\Omega}|\nabla u|^{2} d x \int_{\Omega}\left(2 r \nabla u \nabla \phi-r^{2}|\nabla \phi|^{2}\right) d x\right. \\
& \left.-\left(\int_{\Omega}\left(2 r \nabla u \nabla \phi-r^{2}|\nabla \phi|^{2}\right) d x\right)^{2}\right]
\end{aligned}
$$

that

$$
\begin{aligned}
& \left.\left\langle F_{w}, \phi\right\rangle\right|_{t=1, w=0} \\
& \quad=\lim _{r \rightarrow 0} \frac{F(1,0+r \phi)-F(1,0)}{r} \\
& \quad=-\left(2 a-4 b\|u\|^{2}\right) \int_{\Omega} \nabla u \nabla \phi d x+q \lambda \int_{\Omega}|u|^{p-2} u \phi d x .
\end{aligned}
$$

Consequently, we derive

$$
\left\langle g^{\prime}(0), \phi\right\rangle=-\left.\frac{\left\langle F_{w}, \phi\right\rangle}{F_{t}}\right|_{t=1, w=0}=\frac{\left(2 a-4 b\|u\|^{2}\right) \int_{\Omega} \nabla u \nabla \phi d x-q \lambda \int_{\Omega}|u|^{q-2} u \phi d x}{a(2-q)\|u\|^{2}-b(4-q)\|u\|^{4}} .
$$

This completes the proof. 
Lemma 2.3 If $\lambda \in\left(0, T_{b}\right)$, then we have

(i) the functional $I_{b}$ is coercive and bounded from below on $\Lambda_{b}$;

(ii) $\inf _{\Lambda_{b}^{+} \cup \Lambda_{b}^{0}} I_{b}=\inf _{\Lambda_{b}^{+}} I_{b} \in(-\infty, 0)$.

Proof (i) For $u \in \Lambda_{b}$, by Hölder's inequality, we have

$$
\begin{aligned}
I_{b}(u) & =I_{b}(u)-\frac{1}{4}\left\langle I_{b}^{\prime}(u), u\right\rangle \\
& =\frac{a}{4}\|u\|^{2}-\lambda\left(\frac{1}{q}-\frac{1}{4}\right) \int_{\Omega}|u|^{q} d x \\
& \geq \frac{a}{4}\|u\|^{2}-\lambda\left(\frac{1}{q}-\frac{1}{4}\right)|\Omega|^{\frac{2^{*}-q}{2^{*}}} S^{-q / 2}\|u\|^{q},
\end{aligned}
$$

and the conclusion (i) follows.

(ii) For $u \in \Lambda_{b}^{+}$, there holds

$$
\begin{aligned}
I_{b}(u) & =I_{b}(u)-\frac{1}{q}\left\langle I_{b}^{\prime}(u), u\right\rangle \\
& =a\left(\frac{1}{2}-\frac{1}{q}\right)\|u\|^{2}-b\left(\frac{1}{4}-\frac{1}{q}\right)\|u\|^{4} \\
& <\frac{-a(2-q)\|u\|^{2}+b(4-q)\|u\|^{4}}{4 q}<0 .
\end{aligned}
$$

This together with Lemma 2.1 gives that $\inf _{\Lambda_{b}^{+} \cup \Lambda_{b}^{0}} I_{b}=\inf _{\Lambda_{b}^{+}} I_{b}<0$. Moreover, from (i) we infer that $\inf _{\Lambda_{b}^{+} \cup \Lambda_{b}^{0}} I_{b} \neq-\infty$. Therefore, $\inf _{\Lambda_{b}^{+} \cup \Lambda_{b}^{0}} I \in(-\infty, 0)$.

Lemma 2.4 For all $\lambda>0, I_{b}$ satisfies the $(P S)_{c}$ condition at any level $c<\frac{a^{2}}{4 b}$.

Proof The proof is similar to that of [16, Lemma 2]. We omit the details.

\section{Proof of Theorem 1.1}

Lemma 3.1 Assume that $\lambda \in\left(0, T_{b}\right)$, then problem (1.1) has a positive solution $u_{b}$ with $u_{b} \in \Lambda_{b}^{+}$.

Proof It is easily verified that the sets $\Lambda_{b}^{+} \cup \Lambda_{b}^{0}$ and $\Lambda_{b}^{-}$are closed. Applying the Ekeland variational principle, we can derive a minimizing sequence $\left\{u_{n}\right\} \subset \Lambda_{b}^{+} \cup \Lambda_{b}^{0}$ satisfying that

$$
\lim _{n \rightarrow \infty} I_{b}\left(u_{n}\right)=\inf _{\Lambda_{b}^{+} \cup \Lambda_{b}^{0}} I_{b}<0
$$

and

$$
I_{b}(z) \geq I_{b}\left(u_{n}\right)-\frac{1}{n}\left\|z-u_{n}\right\| \quad \text { for all } z \in \Lambda_{b}^{+} \cup \Lambda_{b}^{0} .
$$

Noting that $I_{b}(|u|)=I_{b}(u)$, we may suppose that $u_{n} \geq 0$ in $\Omega$. By Lemma $2.3,\left\{u_{n}\right\}$ is bounded in $H$, and so we can assume

$$
u_{n} \rightarrow u_{b} \quad \text { in } H
$$




$$
\begin{array}{ll}
u_{n} \rightarrow u_{b} & \text { in } L^{s}(\Omega), 2 \leq s<2^{*} \\
u_{n} \rightarrow u_{b} & \text { a.e. in } \Omega .
\end{array}
$$

In what follows we prove that $u_{b}$ is a positive solution to (1.1). The proof will be divided into four steps.

textbfStep 1: $u_{b} \neq 0$.

By contradiction, we suppose that $u_{b}=0$. Since $u_{n} \in \Lambda_{b}^{+} \cup \Lambda_{b}^{0}$, we see that, for $n$ large,

$$
a\left\|u_{n}\right\|^{2}>\frac{4-q}{2-q} b\left\|u_{n}\right\|^{4} .
$$

As a consequence, we derive

$$
I_{b}\left(u_{n}\right)=\frac{1}{2} a\left\|u_{n}\right\|^{2}-\frac{1}{4} b\left\|u_{n}\right\|^{4}+o_{n}(1) \geq\left(\frac{4-q}{2(2-q)}-\frac{1}{4}\right) b\left\|u_{n}\right\|^{4}+o_{n}(1)>0,
$$

which is a contradiction to (3.1). Thus, $u_{b} \neq 0$.

textbfStep 2: There exists a constant $C_{1}>0$ such that

$$
2 a\left\|u_{n}\right\|^{2}-\lambda(4-q) \int_{\Omega}\left|u_{n}\right|^{q} d x<-C_{1} .
$$

To prove that, it suffices to verify

$$
2 a \liminf _{n \rightarrow \infty}\left\|u_{n}\right\|^{2}<\lambda(4-q) \int_{\Omega}\left|u_{b}\right|^{q} d x .
$$

By $u_{n} \in \Lambda_{b}^{+} \cup \Lambda_{b}^{0}$,

$$
2 a \liminf _{n \rightarrow \infty}\left\|u_{n}\right\|^{2} \leq \lambda(4-q) \int_{\Omega}\left|u_{b}\right|^{q} d x .
$$

Suppose to the contrary that

$$
2 a \liminf _{n \rightarrow \infty}\left\|u_{n}\right\|^{2}=\lambda(4-q) \int_{\Omega}\left|u_{b}\right|^{q} d x .
$$

Then we can assume $\left\|u_{n}\right\|^{2} \rightarrow A>0$ as $n \rightarrow \infty$, where $A$ satisfies

$$
\lambda \int_{\Omega}\left|u_{b}\right|^{q} d x=\frac{2 a A}{4-q} .
$$

Combining this with $\left\{u_{n}\right\} \subset \Lambda_{b}$, we have

$$
0=a A-b A^{2}-\frac{2 a A}{4-q} .
$$

It follows that

$$
A=\frac{a(2-q)}{b(4-q)}
$$


which leads to a contradiction

$$
\begin{aligned}
0 & <\frac{2(2-q)}{(4-q)^{2}} \frac{a^{\frac{4-q}{2}}}{b^{\frac{2-q}{2}}}\left\|u_{n}\right\|^{q}-\lambda \int_{\Omega}\left|u_{n}\right|^{q} d x \\
& \rightarrow \frac{2(2-q)}{(4-q)^{2}} \frac{a^{\frac{4-q}{2}}}{b^{\frac{2-q}{2}}}\left[\frac{a(2-q)}{b(4-q)}\right]^{q / 2}-\frac{2 a}{4-q} \frac{a(2-q)}{b(4-q)} \\
& =\frac{2 a^{2}(2-q)}{b(4-q)^{2}}\left[\left(\frac{2-q}{4-q}\right)^{q / 2}-1\right]<0
\end{aligned}
$$

when $\lambda \in\left(0, T_{b}\right)$. Thus, (3.3) holds.

textbfStep 3: $I_{b}^{\prime}\left(u_{n}\right) \rightarrow 0$ in $H^{-1}$.

Let $0<\rho<\rho_{n} \equiv \rho_{u_{n}}, g_{n} \equiv g_{u_{n}}$, where $\rho_{u_{n}}$ and $g_{u_{n}}$ are given as in Lemma 2.2 with $u=u_{n}$. Let $w_{\rho}=\rho u$ with $\|u\|=1$. Fix $n$ and set $z_{\rho}=g_{n}\left(w_{\rho}\right)\left(u_{n}-w_{\rho}\right)$. By $z_{\rho} \in \Lambda_{b}^{+}$, we have from (3.2) that

$$
I_{b}\left(z_{\rho}\right)-I_{b}\left(u_{n}\right) \geq-\frac{1}{n}\left\|z_{\rho}-u_{n}\right\| .
$$

Then, by the mean value theorem,

$$
\left\langle I_{b}^{\prime}\left(u_{n}\right), z_{\rho}-u_{n}\right\rangle+o\left(\left\|z_{\rho}-u_{n}\right\|\right) \geq-\frac{1}{n}\left\|z_{\rho}-u_{n}\right\| .
$$

Hence, we derive

$$
\left\langle I_{b}^{\prime}\left(u_{n}\right),-w_{\rho}+\left(g_{n}\left(w_{\rho}\right)-1\right)\left(u_{n}-w_{\rho}\right)\right\rangle \geq-\frac{1}{n}\left\|z_{\rho}-u_{n}\right\|+o\left(\left\|z_{\rho}-u_{n}\right\|\right),
$$

and thus,

$$
-\rho\left\langle I_{b}^{\prime}\left(u_{n}\right), u\right\rangle+\left(g_{n}\left(w_{\rho}\right)-1\right)\left\langle I_{b}^{\prime}\left(u_{n}\right), u_{n}-w_{\rho}\right\rangle \geq-\frac{1}{n}\left\|z_{\rho}-u_{n}\right\|+o\left(\left\|z_{\rho}-u_{n}\right\|\right),
$$

from which it follows that

$$
\left\langle I_{b}^{\prime}\left(u_{n}\right), u\right\rangle \leq \frac{1}{n} \frac{\left\|z_{\rho}-u_{n}\right\|}{\rho}+\frac{o\left(\left\|z_{\rho}-u_{n}\right\|\right)}{\rho}+\frac{g_{n}\left(w_{\rho}\right)-1}{\rho}\left\langle I_{b}^{\prime}\left(u_{n}\right), u_{n}-w_{\rho}\right\rangle .
$$

By Step 2, Lemma 2.2, and the boundedness of $\left\{u_{n}\right\}$, one sees that

$$
\left\|z_{\rho}-u_{n}\right\|=\left\|\left(g_{n}\left(w_{\rho}\right)-1\right)\left(u_{n}-w_{\rho}\right)-w_{\rho}\right\| \leq\left|g_{n}\left(w_{\rho}\right)-1\right| C_{2}+\rho
$$

and

$$
\lim _{\rho \rightarrow 0} \frac{\left|g_{n}\left(w_{\rho}\right)-1\right|}{\rho}=\left\langle g_{n}^{\prime}(0), u\right\rangle \leq\left\|g_{n}^{\prime}(0)\right\| \leq C_{3} .
$$

Therefore, for fixed $n$, we deduce by taking $\rho \rightarrow 0$ in (3.4) that

$$
\left\langle I_{b}^{\prime}\left(u_{n}\right), u\right\rangle \leq \frac{C}{n},
$$

which provides that $I_{b}^{\prime}\left(u_{n}\right) \rightarrow 0$ as $n \rightarrow \infty$. 
textbfStep 4: $u_{b}$ is a positive solution of problem (1.1) and $u_{b} \in \Lambda_{b}^{+}$.

It follows from Step 3, Lemmas 2.3 and 2.4 that, along a subsequence, $u_{n} \rightarrow u_{b}$ in $H$ with $I_{b}\left(u_{b}\right)<0$ and $I_{b}^{\prime}\left(u_{b}\right)=0$. Hence, $u_{b} \geq 0$ is a weak solution to problem (1.1) satisfying $u_{b} \in \Lambda_{b}^{+}$. The standard elliptic regularity argument and the strong maximum principle imply that $u_{b}$ is positive. Thus we complete the proof of Lemma 3.1.

Lemma 3.2 Assume that $\lambda \in\left(0, T_{b}\right)$, then problem (1.1) has a positive solution $\tilde{u}_{b}$ with $\tilde{u}_{b} \in \Lambda_{b}^{-}$.

Proof Similar to the proof of Lemma 3.1, one can construct a bounded and nonnegative sequence $\left\{\tilde{u}_{n}\right\} \subset \Lambda_{b}^{-}$satisfying that

(i) $\lim _{n \rightarrow \infty} I_{b}\left(\tilde{u}_{n}\right)=\inf _{\Lambda_{b}^{-}} I_{b}$,

(ii) $\quad I_{b}(z) \geq I_{b}\left(\tilde{u}_{n}\right)-\frac{1}{n}\left\|z-u_{n}\right\|, \quad$ for all $z \in \Lambda_{b}^{-}$,

(iii) $\tilde{u}_{n} \rightarrow \tilde{u}_{b}$ in $H$,

(iv) $\tilde{u}_{n} \rightarrow \tilde{u}_{b}$ in $L^{s}(\Omega), \quad 2 \leq s<2^{*}$,

(v) $\tilde{u}_{n} \rightarrow \tilde{u}_{b} \quad$ a.e. in $\Omega$.

Without loss of generality, we may suppose that $0 \in \Omega$. Take a cut-off function $\varphi(x) \in$ $C_{0}^{\infty}(\Omega)$ satisfying $0 \leq \varphi \leq 1$ in $\Omega$ and $\varphi(x) \equiv 1$ near zero. Define

$$
v_{\varepsilon}(x)=\varphi(x) \frac{(N(N-2))^{(N-2) / 4} \varepsilon^{(N-2) / 2}}{\left(\varepsilon^{2}+|x|^{2}\right)^{1 / 2}} .
$$

It is known that (see [2])

$$
\left\|v_{\varepsilon}\right\|^{2}=S^{N / 2}+O\left(\varepsilon^{N-2}\right) .
$$

Firstly, we prove the following upper bound for $\inf _{\Lambda_{\bar{b}}^{-}} I_{b}$ :

$$
\inf _{\Lambda_{b}^{-}} I_{b}^{-} \leq \sup _{t>0} I_{b}\left(u_{b}+t v_{\varepsilon}\right)<\frac{a^{2}}{4 b}
$$

where $u_{b}$ is the first positive solution obtained in the previous subsection. By $u_{b} \in \Lambda_{b}^{+}$and (1.4), we easily see that $a-b\left\|u_{b}\right\|^{2}>0$. Since $u_{b}$ is a positive solution of (1.1), we also have

$$
0=\left\langle I_{b}^{\prime}\left(u_{b}\right), t v_{\varepsilon}\right\rangle=t\left(a-b\left\|u_{b}\right\|^{2}\right) \int_{\Omega} \nabla u_{b} \nabla v_{\varepsilon} d x-t \lambda \int_{\Omega} u_{b}^{q-1} v_{\varepsilon} d x
$$

from which it follows that

$$
\int_{\Omega} \nabla u_{b} \nabla v_{\varepsilon} d x=\frac{\lambda \int_{\Omega} u_{b}^{q-1} v_{\varepsilon} d x}{a-b\left\|u_{b}\right\|^{2}}>0
$$

To proceed, set $w_{\varepsilon}=u_{b}+R v_{\varepsilon}$ with $R>1$. By (3.7), we have

$$
\left\|w_{\varepsilon}\right\|^{2}=\left\|u_{b}\right\|^{2}+2 R \int_{\Omega} \nabla u_{b} \nabla v_{\varepsilon} d x+R^{2}\left\|v_{\varepsilon}\right\|^{2} \geq\left\|u_{b}\right\|^{2}+R^{2} S^{3 / 2}+O(\varepsilon) .
$$


Let $h(t)$ be defined as in Lemma 2.1. As can be seen from the proof of Lemma 2.1, we have that $h\left(t_{\varepsilon}\right)=\lambda \int_{\Omega}\left|\frac{w_{\varepsilon}}{\left\|w_{\varepsilon}\right\|}\right|^{q} d x$ and $h^{\prime}\left(t_{\varepsilon}\right)<0$, where $t_{\varepsilon}=t^{+}\left(\frac{w_{\varepsilon}}{\left\|w_{\varepsilon}\right\|}\right)$. From the structure of $h$ and $\int_{\Omega}\left|\frac{w_{\varepsilon}}{\left\|w_{\varepsilon}\right\|}\right|^{q} d x>0$, it follows that $t_{\varepsilon}$ is uniformly bounded by a suitable positive constant $C_{1}$, $\forall R \geq 1$ and $\forall \varepsilon>0$.

On the other hand, we can infer from (3.8) that there exists $\varepsilon_{1}>0$ such that

$$
\left\|w_{\varepsilon}\right\|^{2} \geq\left\|u_{b}\right\|^{2}+\frac{1}{2} R^{2} S^{3 / 2}, \quad \forall \varepsilon \in\left(0, \varepsilon_{1}\right) .
$$

Thus, we can find $R_{1} \geq 1$ such that $\left\|w_{\varepsilon}\right\|>C_{1}, \forall R \geq R_{1}$, and $\forall \varepsilon \in\left(0, \varepsilon_{1}\right)$.

Let

$$
E_{1}=\left\{u: u=0 \text { or }\|u\|<t^{+}\left(\frac{u}{\|u\|}\right)\right\} \quad \text { and } \quad E_{2}=\left\{u:\|u\|>t^{+}\left(\frac{u}{\|u\|}\right)\right\} .
$$

Note that $H-\Lambda_{b}^{-}=E_{1} \cup E_{2}$ and $\Lambda_{b}^{+} \subset E_{1}$. Since $u_{b} \in \Lambda_{b}^{+}$, by the continuity of $t^{+}(u)$, one sees that $u_{b}+t R_{1} v_{\varepsilon}$ for $t \in(0,1)$ must intersect $\Lambda_{b}^{-}$, and consequently

$$
\inf _{\Lambda_{b}^{-}} I_{b} \leq \sup _{t>0} I_{b}\left(u_{b}+t v_{\varepsilon}\right) .
$$

Hence (3.5) will follow if we show that

$$
\sup _{t>0} I_{b}\left(u_{b}+t v_{\varepsilon}\right)<\frac{a^{2}}{4 b}
$$

By the mean value theorem, we can get $\delta(x) \in[0,1]$ satisfying

$$
\left(u_{b}(x)+t v_{\varepsilon}(x)\right)^{q}-u_{b}^{q}(x)=q\left(u_{b}(x)+\delta(x) t v_{\varepsilon}(x)\right)^{q-1} t v_{\varepsilon}(x) \geq q t u_{b}^{q-1}(x) v_{\varepsilon}(x)
$$

for any $x \in \Omega$. By (3.6), (3.7), and (3.9),

$$
\begin{aligned}
& I_{b}\left(u_{b}+t v_{\varepsilon}\right) \\
&=\frac{a}{2}\left\|u_{b}\right\|^{2}+a t \int_{\Omega} \nabla u_{b} \nabla v_{\varepsilon} d x+\frac{a}{2} t^{2}\left\|v_{\varepsilon}\right\|^{2}-\frac{b}{4}\left\|u_{b}\right\|^{4}-b t^{2}\left(\int_{\Omega} \nabla u_{b} \nabla v_{\varepsilon} d x\right)^{2} \\
& \quad-\frac{b}{4} t^{4}\left\|v_{\varepsilon}\right\|^{4}-b t\left\|u_{b}\right\|^{2} \int_{\Omega} \nabla u_{b} \nabla v_{\varepsilon} d x-\frac{b}{2} t^{2}\left\|u_{b}\right\|^{2}\left\|v_{\varepsilon}\right\|^{2} \\
& \quad-b t^{3}\left\|v_{\varepsilon}\right\|^{2} \int_{\Omega} \nabla u_{b} \nabla v_{\varepsilon} d x-\frac{\lambda}{q} \int_{\Omega}\left(u_{b}+t v_{\varepsilon}\right)^{q} d x \\
& \leq I_{b}\left(u_{b}\right)+\frac{a}{2} t^{2}\left\|v_{\varepsilon}\right\|^{2}-\frac{b}{4} t^{4}\left\|v_{\varepsilon}\right\|^{4}-\frac{b}{2} t^{2}\left\|u_{b}\right\|^{2}\left\|v_{\varepsilon}\right\|^{2} \\
& \quad-\frac{\lambda}{q} \int_{\Omega}\left[\left(u_{b}+t v_{\varepsilon}\right)^{q}-u_{b}^{q}-q t u_{b}^{q-1} v_{\varepsilon}\right] d x \\
& \leq \frac{a}{2} t^{2}\left\|v_{\varepsilon}\right\|^{2}-\frac{b}{4} t^{4}\left\|v_{\varepsilon}\right\|^{4}-\frac{b}{2} t^{2}\left\|u_{b}\right\|^{2}\left\|v_{\varepsilon}\right\|^{2}
\end{aligned}
$$

which implies that there exists $t_{1}>0$ small enough such that

$$
\sup _{0<t<t_{1}} I_{b}\left(u_{b}+t v_{\varepsilon}\right)<\frac{a^{2}}{4 b} .
$$


Thus, we only need to consider the case of $t \geq t_{1}$. Since

$$
\begin{aligned}
\sup _{t \geq t_{1}} I_{b}\left(u_{b}+t v_{\varepsilon}\right) & \leq \sup _{t>0}\left\{\frac{a}{2} t^{2}\left\|v_{\varepsilon}\right\|^{2}-\frac{b}{4} t^{4}\left\|v_{\varepsilon}\right\|^{4}\right\}-\frac{b}{2} t_{1}^{2}\left\|u_{b}\right\|^{2}\left\|v_{\varepsilon}\right\|^{2} \\
& =\frac{a^{2}}{4 b}-\frac{b}{2} t_{1}^{2}\left\|u_{b}\right\|^{2}\left\|v_{\varepsilon}\right\|^{2}<\frac{a^{2}}{4 b}
\end{aligned}
$$

we deduce that (3.5) holds.

Secondly, we claim that $\tilde{u}_{b} \neq 0$. If, to the contrary, $\tilde{u}_{b}=0$, from $\left\{\tilde{u}_{n}\right\} \subset \Lambda_{b}^{-}$it then follows that

$$
a\left\|\tilde{u}_{n}\right\|^{2}-b\left\|\tilde{u}_{n}\right\|^{4}+o_{n}(1)=0
$$

As a consequence, we obtain $\left\|\tilde{u}_{n}\right\|^{2} \rightarrow \frac{a}{b}$ as $n \rightarrow \infty$. Furthermore,

$$
\inf _{\Lambda_{b}^{-}} I_{b}=\lim _{n \rightarrow \infty} I_{b}\left(\tilde{u}_{n}\right)=\lim _{n \rightarrow \infty}\left[\frac{a}{2}\left\|\tilde{u}_{n}\right\|^{2}-\frac{b}{4}\left\|\tilde{u}_{n}\right\|^{4}-\lambda \int_{\Omega}\left|\tilde{u}_{n}\right|^{q} d x\right]=\frac{a^{2}}{4 b}
$$

which contradicts (3.5). Hence, the claim holds. This time we can proceed as in the proof of Lemma 3.1 and deduce that $\tilde{u}_{b}$ is a positive solution of problem (1.1) with $\tilde{u}_{b} \in \Lambda_{b}^{-}$. The proof is complete.

Proof of Theorem 1.1 This is an immediate consequence of (1.4), Lemmas 3.1 and 3.2.

\section{Proof of Theorem 1.2}

Proof of Theorem 1.2 For any sequence $\left\{b_{n}\right\}$ with $b_{n} \searrow 0$, we can use Theorem 1.1 to obtain sequences $\left\{u_{b_{n}}\right\} \subset \Lambda_{b_{n}}^{+}$and $\left\{\tilde{u}_{b_{n}}\right\} \subset \Lambda_{b_{n}}^{-}$corresponding to positive solutions to problem (1.1) with $b=b_{n}$ when $\lambda \in\left(0, T_{b_{n}}\right)$.

By $\tilde{u}_{b_{n}} \in \Lambda_{b_{n}}^{-}$and (1.4), we see that

$$
\lim _{n \rightarrow \infty}\left\|\tilde{u}_{b_{n}}\right\|^{2} \geq \lim _{n \rightarrow \infty} \frac{a(2-q)}{b_{n}(4-q)}=\infty
$$

and conclusion (i) follows.

Next, we prove conclusion (ii) of Theorem 1.2. Note that

$$
I_{b}\left(u_{b_{n}}\right)=\inf _{\Lambda_{b_{n}}^{+} \cup \Lambda_{b_{n}}^{0}} I_{b_{n}}<0
$$

for all $n \in \mathbb{N}$. Then, by Hölder's inequality, we have

$$
\begin{aligned}
0 & \geq I_{b_{n}}\left(u_{b_{n}}\right)-\frac{1}{4}\left\langle I_{b_{n}}^{\prime}\left(u_{b_{n}}\right), u_{b_{n}}\right\rangle \\
& \geq\left(\frac{1}{2}-\frac{1}{4}\right)\left\|u_{b_{n}}\right\|^{2}-\lambda\left(\frac{1}{q}-\frac{1}{4}\right)|\Omega|^{\frac{2^{*}-q}{2^{*}}} S^{\frac{-q}{2}}\left\|u_{b_{n}}\right\|^{q} .
\end{aligned}
$$

Since $1<q<2$, it follows that $\left\{u_{b_{n}}\right\}$ is bounded in $H$. As a consequence, there exists a subsequence of $\left\{b_{n}\right\}$ (still denoted by $\left\{b_{n}\right\}$ ) such that $u_{b_{n}} \rightarrow u_{0}$ in $H$ as $n \rightarrow \infty$. Furthermore, 
we have that, for all $v \in H$,

$$
\begin{aligned}
0 & =\lim _{n \rightarrow \infty}\left\langle I_{b_{n}}^{\prime}\left(u_{b_{n}}\right), v\right\rangle \\
& =\lim _{n \rightarrow \infty}\left[\left(a-b_{n}\left\|u_{b_{n}}\right\|^{2}\right) \int_{\Omega} \nabla u_{b_{n}} \nabla v d x-\lambda \int_{\Omega} u_{b_{n}}^{q-1} v d x\right] \\
& =a \int_{\Omega} \nabla u_{0} \nabla v d x-\lambda \int_{\Omega} u_{0}^{q-1} v d x,
\end{aligned}
$$

which implies that $u_{0}$ is a positive solution to problem (1.5). To complete the proof, we only need to show that $u_{b_{n}} \rightarrow u_{0}$ in $H$. This follows easily from

$$
\begin{aligned}
& a\left\|u_{b_{n}}-u_{0}\right\|^{2} \\
& =\left\langle I_{b_{n}}^{\prime}\left(u_{b_{n}}\right)-I_{0}^{\prime}\left(u_{0}\right), u_{b_{n}}-u_{0}\right\rangle+b_{n} \int_{\Omega}\left|\nabla u_{b_{n}}\right|^{2} d x \int_{\Omega} \nabla u_{b_{n}} \nabla\left(u_{b_{n}}-u_{0}\right) d x \\
& \quad+\lambda \int_{\Omega}\left(u_{b_{n}}^{q-1}-u_{0}^{q-1}\right)\left(u_{b_{n}}-u_{0}\right) d x \\
& \quad \rightarrow 0,
\end{aligned}
$$

as $n \rightarrow \infty$. Theorem 1.2 is thus proved.

\section{Acknowledgements}

The authors would like to thank the referees for their careful reading of this paper and for the valuable comments.

\section{Funding}

Not applicable.

\section{Availability of data and materials}

Not applicable.

\section{Competing interests}

The authors declare that they have no competing interests.

\section{Authors' contributions}

Both authors contributed equally and significantly in this paper. Both authors read and approved the final manuscript.

\section{Author details}

${ }^{1}$ Teaching and Research Department of Mathematics and Physics, Fujian Jiangxia University, Fuzhou, 350108, P.R. China.

${ }^{2}$ Department of Basic Teaching and Research, Yango University, Fuzhou, 350015, P.R. China.

\section{Publisher's Note}

Springer Nature remains neutral with regard to jurisdictional claims in published maps and institutional affiliations.

Received: 10 November 2020 Accepted: 18 May 2021 Published online: 26 May 2021

\section{References}

1. Alves, C.O., Corrêa, F.J.S.A., Ma, T.F.: Positive solutions for a quasilinear elliptic equation of Kirchhoff type. Comput. Math. Appl. 49, 85-93 (2005)

2. Brézis, H., Nirenberg, L.: Positive solutions of nonlinear elliptic problems involving critical Sobolev exponent. Commun. Pure Appl. Math. 36, 437-477 (1983)

3. Duan, Y., Sun, X., Li, H.Y.: Existence and multiplicity of positive solutions for a nonlocal problem. J. Nonlinear Sci. Appl. $10,6056-6061(2017)$

4. Gan, W., Liu, S.: Multiple positive solutions of a class of Schrödinger-Poisson equation involving indefinite nonlinearity in $\mathbb{R}^{3}$. Appl. Math. Lett. 93,111-116 (2019)

5. He, X., Zou, W.: Existence and concentration behavior of positive solutions for a Kirchhoff equation in $\mathbb{R}^{3}$. J. Differ. Equ. $252,1813-1834(2012)$

6. Lei, C., Chu, C., Suo, H.: Positive solutions for a nonlocal problem with singularity. Electron. J. Differ. Equ. 2017, 85 (2017) 
7. Lei, C., Liao, J., Suo, H.: Multiple positive solutions for a class of nonlocal problems involving a sign-changing potential. Electron. J. Differ. Equ. 2017, 9 (2017)

8. Liu, X., Hu, G., Huang, G., Sun, C.: An elastic metamaterial with simultaneously negative mass density and bulk modulus. Appl. Phys. Lett. 98, 1-3 (2011)

9. Naimen, D.: The critical problem of Kirchhoff type elliptic equations in dimension four. J. Differ. Equ. 257, 1168-1193 (2014)

10. Qian, X., Chao, W.: Existence of positive solutions for nonlocal problems with indefinite nonlinearity. Bound. Value Probl. 2020, 40 (2020)

11. Qian, X., Chen, J.: Existence of multiple solutions and estimates of extremal values for a Kirchhoff type problem with fast increasing weight and critical nonlinearity. Electron. J. Differ. Equ. 2018, 144 (2018)

12. Sun, Y., Li, S.: A nonlinear elliptic equation with critical exponent: estimates for extremal values. Nonlinear Anal. 69 , 1856-1869 (2008)

13. Tarantello, G.: On nonhomogeneous elliptic equations involving critical Sobolev exponent. Ann. Inst. Henri Poincaré, Anal. Non Linéaire 9, 281-304 (1992)

14. Wang, Y., Suo, H., Lei, C.: Multiple positive solutions for a nonlocal problem involving critical exponent. Electron. J. Differ. Equ. 2017, 275 (2017)

15. Wang, Y., Yang, X.: Infinitely many solutions for a new Kirchhoff-type equation with subcritical exponent. Appl. Anal. 4 , $1-14(2020)$

16. Yin, G., Liu, J.: Existence and multiplicity of nontrivial solutions for a nonlocal problem. Bound. Value Probl. 2015, 26 (2015)

17. Zhang, J., Zhang, Z:: Existence of nontrivial solution for a nonlocal problem with subcritical nonlinearity. Adv. Differ. Equ. 2018, 359 (2018)

Submit your manuscript to a SpringerOpen ${ }^{\circ}$ journal and benefit from:

- Convenient online submission

- Rigorous peer review

- Open access: articles freely available online

- High visibility within the field

- Retaining the copyright to your article

Submit your next manuscript at $\boldsymbol{\nabla}$ springeropen.com 